According to our results, self-reported adherence appears to be a cost and time effective method to care for patients.

References:

[1] Monchablon C, et. al. Assessment adherence to disease-modifying anti-rheumatic drugs in rheumatoid arthritis. Clinical Rheumatology. 2019;39(1):207-216.

[2] Scott DL, et al. (2010) Rheumatoid arthritis. Lancet 376:1094-1108.

Disclosure of Interests: None declared

DOI: 10.1136/annrheumdis-2020-eular.6447

\section{AB1209 $\quad$ A SYSTEMATIC REVIEW ON THE EFFECT OF DMARDS ON FERTILITY IN RHEUMATOID ARTHRITIS}

L. Brants ${ }^{1}$, I. Soenen ${ }^{1}$, S. Pazmino ${ }^{1}$, R. Westhovens ${ }^{1,2}$, P. Verschueren ${ }^{1,2}$, D. De Cock'. 'Skeletal Biology and Engineering Research Centre, KU Leuven, Leuven, Belgium; ${ }^{2}$ Rheumatology, University Hospitals Leuven, Leuven, Belgium

Background: Patients with rheumatoid arthritis (RA) seem to experience a diminished fertility. Reasons for this lowered fertility are insufficiently defined and probably multifactorial. Although the effect of DMARDs on pregnancy outcomes have been studied, there is a lack of data on the effect of DMARDs on the fertility of patients with RA.

Objectives: To evaluate all studies that concern an effect of DMARDs on the fertility of men and women with RA in a systematic review.

Methods: A search was conducted at 18/10/2019 in three databases including Embase, Pubmed (Medline) and Web Of Science with specific search strings for each database, constructed with the help from a health sciences librarian. We included studies involving women or men diagnosed with RA, of fertile age (1845years) and on a DMARD therapy, with as outcome a fertility parameter. Systematic reviews, meta-analyses, case reports, case series and animal studies were excluded. Studies not in English or Dutch or written more than 15 years ago were excluded. Article selection was firstly based on title/abstract (double blind, two researchers, LB and IS) and then full text (two researchers, LB and IS). In case consensus could not be reached, a third researcher (DDC) was consulted. The references of included articles were reviewed ("snowballing") to include and minimize the missing articles. A quality check of the included full text papers was performed using the CASP Appraisal Checklists. A chart was made based on outcomes of interest.

Results: After duplicate removal, 9030 articles were found. After title/abstract screening, 82 articles remained. After full text screening, 4 articles could be retained. No additional studies were found through snowballing. Only studies about women could be included, as the evidence found for men was all in papers with exclusion criteria for our systematic review (e.g. case reports). Table 1 summarizes these papers. The included studies investigated the following DMARDs: methotrexate (MTX), certolizumab pegol (CZP), etanercept (ETN) and sulfasalazine (SSZ). No detrimental effects of these DMARDs on fertility, defined as timeto-pregnancy (TTP), anti-Müllerian hormone serum level or presence of a history of infertility, were reported.

Conclusion: This systematic review underlines the knowledge gap on the effect of DMARDs on fertility in human studies. Only 4 studies on women, and no studies on men were found. In the 4 included studies, DMARD treatment, even with MTX in contrast to general belief, had no harmful effect on fertility, probably because disease activity was better controlled with DMARD therapy. However, effects of other RA medication such as NSAIDs were excluded. More research is needed to improve guidance for patients with RA with a child wish.
Disclosure of Interests: Liesbeth Brants: None declared, Isaline Soenen: None declared, Sofia Pazmino: None declared, Rene Westhovens Grant/research support from: Celltrion Inc, Galapagos, Gilead, Consultant of: Celltrion Inc, Galapagos Gilead, Speakers bureau: Celltrion Inc, Galapagos, Gilead, Patrick Verschueren Grant/research support from: Pfizer unrestricted chair of early RA research, Speakers bureau: various companies, Diederik De Cock: None declared DOI: 10.1136/annrheumdis-2020-eular.2856

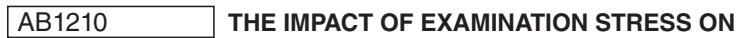 AUTOIMMUNE DISEASES AMONG UNIVERSITY STUDENTS}

F. Fayed ${ }^{1}$, E. Abdelkarim ${ }^{1}$, M. Morsy ${ }^{2} .{ }^{1}$ Alexandria University Student Hospital, Rheumatology and Immunology Unit, Alexandria, Egypt; ${ }^{2}$ Alexandria University Main Hospital, Alexandria, Egypt

Background: Stress is a risk factor of various diseases including autoimmune diseases. Autoimmune diseases are one of the leading causes of morbidity in young adults. ${ }^{(1)}$ Examination stress is a main concern nowadays due to the study style, lack of preparation, doctor- student relationship and family pressure. ${ }^{(2)}$ The previous studies declared that stress may causes neuroendocrinal changes leading to immune dysregulations and cytokines production. ${ }^{(3)}$

Objectives: The aim of study is to scope the light on the importance of stress as a predisposing factor in autoimmune disease flares particularly Examination stress. Methods: A three-year (2017-2019) cross-sectional prospective study conducted on 1365 students who presented to the Alexandria University rheumatology clinic during examinations. Clinical assessments, routine investigations, activity markers, activity indices, stress and anxiety questionnaires and perceived stress scale (PSS) were applied to all patients during consecutive visits.

Results: Through 5800 visits in three years during examination sessions, patients age ranged from ( $17-25)$ years with $76 \%$ females and $24 \%$ males. They grouped into SLE (31.35\%), Rheumatoid arthritis (RA) (37.28\%), Fibromyalgia (13.91\%), FMF (2.63\%), Ankylosing Spondylitis (1.75\%), Psoriatic arthritis (0.73\%), systemic sclerosis $(0.58 \%)$, and undifferentiated connective tissue (11.73\%). According to SLE patients, $43.92 \%$ were newly diagnosed whilst $54.16 \%$ of previously diagnosed SLE presented with Flare in particular lupus nephritis $(56.33 \%)$, arthritis $(43.22 \%)$, hematological $(49.76 \%)$ and serositis $(21.36 \%)$. Interestingly, RA patients who newly diagnosed were $35.16 \%$ of total RA patients while $42.42 \%$ of previously diagnosed RA patients presented with moderate and high DAS-28 due to incompliance with treatment in $(64.37 \%)$ of patients, $(11.53 \%$ on biological, $88.47 \%$ on conventional treatment). In addition, (49.36\%) of FMF presented in recent attacks. It was also found that Arthralgia, bone aches and sleep deprivation are the main complaints. Concerning, A High perceived stress scale (PSS) was associated with High DAS28 and SLEDI-2K scores. $(r=0.723,0.865)(P<0.001)$ Conclusion: Examination stress is one of triggering factor for autoimmune disease flares. It is associated with high disease activities and ruthless outcomes. References:

[1] Cooper, G. S., \& Stroehla, B. C. The epidemiology of autoimmune diseases Autoimmunity Reviews,2003:2(3);119-25.

[2] Archana kumari, jagrati jain. Examination stress and anxiety: a study of college students. Global Journal of Multidisciplinary Studies 2014:4:ISSN 2348-0459

Disclosure of Interests: None declared

DOI: 10.1136/annrheumdis-2020-eular.4681

\begin{tabular}{l|l}
\hline AB1211 & IMMUNE-RELATED ADVERSE EVENTS IN PATIENTS \\
RECEIVING PD-1/PD-L1 INHIBITORS: PRELIMINARY \\
RESULTS FROM A PROSPECTIVE COHORT STUDY
\end{tabular}

A. Ferrarini ${ }^{1}$, D. Benfaremo ${ }^{1}$, G. Rossetti ${ }^{1}$, F. Morgese ${ }^{2}$, C. Tonnini ${ }^{1}$, R. Berardi ${ }^{2}$, A. Gabrielli', G. Pomponio'. ${ }^{1}$ Università Politecnica delle Marche, Clinica

Table 1. Characteristics of studies included in the systematic review

\begin{tabular}{|c|c|c|c|c|c|c|c|}
\hline Authors & Location & Sample & DMARD & Outcome & Method & Design & Result \\
\hline $\begin{array}{l}\text { Akintayo et al. } \\
2018\end{array}$ & Nigeria & $\begin{array}{c}50 \text { women with RA and } \\
50 \text { women without } \\
\text { RA }\end{array}$ & MTX & $\begin{array}{l}\text { Infertility or history of } \\
\text { infertility }\end{array}$ & $\begin{array}{c}\text { Interviewer-administered } \\
\text { questionnaire }\end{array}$ & Retrospective study & $\begin{array}{c}\text { MTX was associated with } \\
\text { a negative history of } \\
\text { infertility }\end{array}$ \\
\hline $\begin{array}{l}\text { Shimada et al. } \\
2019\end{array}$ & Japan & $\begin{array}{c}25 \text { pregnancies in } 19 \\
\text { patients with RA }\end{array}$ & CZP and ETN & TTP (time to pregnancy) & medical records & Retrospective study & $\begin{array}{c}\text { bDMARD treatment short- } \\
\text { ened the TTP }\end{array}$ \\
\hline $\begin{array}{l}\text { Brouwer et al. } \\
2013\end{array}$ & The Netherlands & $\begin{array}{l}72 \text { women with } \\
\text { recent-onset RA } \\
\text { compared to } 509 \\
\text { healthy women }\end{array}$ & MTX & Level of serum AMH & $\begin{array}{l}\text { medical records, serum } \\
\text { samples ( } 2 \text { time points) }\end{array}$ & Retrospective study & $\begin{array}{c}\text { AMH levels were not lower } \\
\text { with MTX. }\end{array}$ \\
\hline $\begin{array}{l}\text { Brouwer et al. } \\
2014\end{array}$ & The Netherlands & 245 women with RA & MTX and SSZ & TTP & $\begin{array}{c}\text { Questionnaires and } \\
\text { interviews }\end{array}$ & Prospective cohort study & $\begin{array}{l}\text { MTX and SSZ did not } \\
\text { prolong TTP }\end{array}$ \\
\hline
\end{tabular}

RA = Rheumatoid Arthritis; MTX = Methotrexate; TNFi = Tumor Necrosis Factor inhibitor; CZP = Certolizumab pegol; ETN = Etanercept; SSZ = Sulfasalazine; TTP = Time to Pregnancy; DMARD = Disease Modifying Antirheumatic Drug; AMH = Anti-Müllerian hormone 
Medica, Dipartimento di Scienze Cliniche e Molecolari, Ancona, Italy ${ }^{2}$ Università Politecnica delle Marche, Clinica Oncologica, Dipartimento Scienze Cliniche e Molecolari, Ancona, Italy

Background: Recent introduction of immune checkpoint inhibitors (ICls) revolutionized oncological guidelines. Immune-related adverse events (IrAEs) may occur in as many as $85 \%$ of patients (10\% with toxicity grade $3 / 4)$, but detailed epidemiology of irAEs is still lacking, mostly because of data collection and analysis vary widely.

Objectives: The purpose of our study is to establish a prospective cohort of patients treated with PD-1/PD-L1 inhibitors in order to determine incidence, risk factors and characteristics of irAEs in a real-world setting

Methods: We conducted a prospective cohort study enrolling patients receiving anti-PD-1/PD-L1 agents for the treatment of metastatic or locally advanced nonsmall cell lung cancer, renal cell carcinoma, squamous cell carcinoma of the head and neck, Hodgkin lymphoma. Detailed recommendations have been implemented for cases fulfilling criteria for suspected irAEs, including procedures for evaluation and diagnosis, specific treatments and rules for drug discontinuation. IrAEs have been defined and graded according to Common Terminology Criteria for Adverse Events vs 5.0 . Management strategies have been adapted by a multidisciplinary panel, basing on the oncological guidelines, which represent the current best clinical practice. AEs screening, physical examination, ECG and clinical laboratory evaluation have been performed at baseline visit and follow up (4, 8, 12 weeks). Results: Fifty-two patients have been enrolled from Jan 2019 to Dec 2020. Characteristics are reported in the Table below. Twelve patients developed irAEs (23\%), 6 treated with nivolumab, 4 with pembrolizumab, 1 with atezolizumab and 1 with durvalumab. Mild-to-moderate (G1-G2) irAEs were hepatitis, hypothyroidism, III-V-VII cranial nerve palsy, polymyalgia-like syndrome, skin psoriasis and type-1 diabetes mellitus. Severe cases (G3) of bullous dermatitis, Lichen Planus, interstitial pneumonia and myositis occurred. One patient developed three different irAEs. Median time of onset was 4.5 weeks. IrAEs were successfully treated according to established guideline, but 4 patients stopped anti-neoplastic therapy due to irAEs and 11 for disease progression. Five patients died.

Conclusion: Cancer patients receiving PD-1/PD-L1 agents are being prospectively followed. Preliminary results confirm that $1 / 4$ patients may develop irAEs. Innovative tools are required in order to manage irAEs, prevent potential relapse and avoid useless interruption of therapy. Further research needs to get insights into pathophysiological mechanisms and risk factors.

References:

[1] Arnaud-Coffin P. A systematic review of adverse events in randomized trials assessing immune checkpoint inhibitors. Int J Cancer. 2019; Champiat S, Lambotte O, Barreau E, et al. Management of immune checkpoint blockade dysimmune toxicities: a collaborative position paper. Annals of Oncology 27, 2016.

\begin{tabular}{lc}
\hline Characteristics & $\mathbf{N}=\mathbf{5 2}$ \\
\hline Age [years, median (range)] & $67(31-92)$ \\
Gender (F/M) & $17 / 35$ \\
Cancer type & 12 \\
Melanoma & 9 \\
Renal cell carcinoma & 29 \\
Non-small-cell lung carcinoma Hodgkin & 1 \\
lymphoma & 1 \\
Head-neck cancer & \\
Agents & 24 \\
Nivolumab & 16 \\
Pembrolizumab & 8 \\
Atezolizumab & 4 \\
Durvalumab & $\mathbf{1 2} \mathbf{p t s}(\mathbf{2 3} \%)^{*}$ \\
irAEs & 4 \\
G1 & 6 \\
G2 & 4 \\
G3 & 0 \\
G4 & $\mathbf{2 0}(\mathbf{3 9 \%})$ \\
Drug discontinuation & 4 \\
due to irAEs & 11 \\
due to disease progression & 5 \\
death & \\
\hline
\end{tabular}

*one patient had more than one event

Disclosure of Interests: None declared

DOI: 10.1136/annrheumdis-2020-eular.2850

\section{AB1212 ATHEROSCLEROSIS IN PATIENTS WITH PSORIATIC ARTHRITIS VS RHEUMATOID ARTHRITIS: A CASE CONTROL STUDY}

D. Á. Galarza-Delgado ${ }^{1}$, J. R. Azpiri-López ${ }^{2}$, I. J. Colunga-Pedraza ${ }^{1}$, D.

E. Flores Alvarado ${ }^{1}$, O. Ilizaliturri Guerra ${ }^{1}$, P. F. Frausto Lerma ${ }^{1}$, A. Pérez
Villar ${ }^{1}$, M. A. Reyes Soto ${ }^{1}$, I. C. Zárate Salinas ${ }^{2}$, A. C. Garza Acosta ${ }^{3} .{ }^{1}$ Hospital Universitario Dr. José Eleuterio González, Rheumatology Department, Monterrey, Mexico; ${ }^{2}$ Hospital Universitario Dr. José Eleuterio González, Cardiology Department, Monterrey, Mexico; ${ }^{3}$ Hospital Universitario Dr. José Eleuterio González, Radiology Department, Monterrey, Mexico

Background: Rheumatic diseases such as Psoriatic Arthritis (PsA) and Rheumatoid Arthritis (RA) are associated with increased morbidity and mortality, mainly due to cardiovascular causes. Cardiovascular outcomes in patients with PsA and RA cannot be completely explained by traditional cardiovascular risk factors, suggesting that the systemic inflammation that characterizes these diseases may have an important role on accelerated atherosclerosis. ${ }^{1}$

Objectives: To compare carotid intima-media thickness (cIMT) and asymptomatic carotid plaque $(\mathrm{CP})$ prevalence, between patients with $\mathrm{PsA}, \mathrm{RA}$ and controls.

Methods: Cross-sectional observational study. Seventy patients, aged 35-75 years, with PSA and RA who fulfilled the CASPAR and ACR/EULAR 2010 classification criteria, respectively, who were active on a cardio-rheuma preventive clinic were recruited, matched with 70 healthy controls. All groups underwent a noninvasive examination using B-mode ultrasonography of the right and left common carotid artery. CP was defined as a focal narrowing $\geq 0.5 \mathrm{~mm}$ of the surrounding lumen or cIMT $\geq 1.2 \mathrm{~mm}$; hyperplasia of the carotid intima was defined as cIMT $\geq 0.9 \mathrm{~mm}$ to $1.1 \mathrm{~mm}$. Descriptive data were analyzed by continuous and categorical variables. Continuous variables with normal distribution are shown as mean \pm standard deviation (SD), and non-normal distribution as median and quartiles (25q-75q). ANOVA, Kruskal Wallis, $X^{2}$ and Mann-Whitney $U$ were used to compared data. A $p$ value $\leq 0.05$ was considered statistically significant. Statistical analysis was done using SPSS version 24 (IBM Corp., Armonk, NY, USA). Results: Clinical and demographic characteristics are shown in Table 1. The global prevalence of carotid atherosclerosis was $25.7 \%$ and $38.6 \%$ in RA and PsA respectively, and $27.1 \%$ in controls $(p=0.170)$. Intimal hyperplasia was found in $20 \%, 12.9 \%$ and $0 \%$ in RA, PsA and controls $(p=0.001)$, respectively (Table 2$)$

Table 1. Clinical and demographic characteristics

\begin{tabular}{lcccc}
\hline & $\begin{array}{c}\mathbf{R A} \\
\mathbf{n = 7 0}\end{array}$ & $\begin{array}{c}\text { PsA } \\
\mathbf{n = 7 0}\end{array}$ & $\begin{array}{c}\text { Controls } \\
\mathbf{n = 7 0}\end{array}$ & $\begin{array}{c}P \\
\text { value }\end{array}$ \\
\hline $\begin{array}{l}\text { Gender } \\
\text { Male }\end{array}$ & $9(12.9 \%)$ & $31(44.4 \%)$ & $11(15.7 \%)$ & $<\mathbf{0 . 0 0 1}$ \\
$\quad$ Female & $61(87.1 \%)$ & $39(55.7 \%)$ & $59(84.3 \%)$ & \\
Age, years & $54.51 \pm 9.657$ & $53.1 \pm 10.87$ & $53.54 \pm 7.48$ & NS \\
Body Mass Index, BMI, & $28.99(25.95-32.34)$ & 29.04 & 27.44 & NS \\
$\quad$ kg/m ${ }^{2}$ & & $(26.23-31.92)$ & $(24.98-30.95)$ & \\
Comorbidities & & & & \\
$\quad$ Diabetes Mellitus & $11(15.7 \%)$ & $14(20 \%)$ & $8(11.4 \%)$ & NS \\
$\quad$ Hypertension & $24(34.3 \%)$ & $27(38.6 \%)$ & $15(21.4 \%)$ & NS \\
$\quad$ Dyslipidemia & $19(27.1 \%)$ & $30(42.9 \%)$ & $18(25.7 \%)$ & NS \\
$\quad$ Active smoker & $7(10 \%)$ & $16(22.9 \%)$ & $15(21.4 \%)$ & NS \\
Disease duration & & & & \\
$\quad$ Duration, years & $8.45(3.34-15.88)$ & $5(2.75-8)$ & - & $\mathbf{0 . 0 0 5}$ \\
Statins & $9(12.9 \%)$ & $12(17.1 \%)$ & $10(14.3 \%)$ & NS \\
\hline
\end{tabular}

Table 2. Ultrasonographic characteristics

\begin{tabular}{lcccc}
\hline & $\begin{array}{c}\text { RA } \\
\mathbf{n}=\mathbf{7 0}\end{array}$ & $\begin{array}{c}\text { PsA } \\
\mathbf{n}=\mathbf{7 0}\end{array}$ & $\begin{array}{c}\text { Controls } \\
\mathbf{n}=\mathbf{7 0}\end{array}$ & $P$ value \\
\hline Right CIMT, mm & $0.8(0.6-1.1)$ & $0.6(0.5-0.9)$ & $0.6(0.5-0.8)$ & $<0.001$ \\
Left CIMT, mm & $0.8(0.6-0.9)$ & $0.6(0.5-0.7)$ & $0.6(0.5-1.2)$ & $\mathbf{0 . 0 0 2}$ \\
Any Hyperplasia & $14(20 \%)$ & $9(12.9 \%)$ & 0 & $\mathbf{0 . 0 0 1}$ \\
Right intimal hyperplasia & $7(10 \%)$ & $5(7.1 \%)$ & 0 & $\mathbf{0 . 0 2 1}$ \\
Left intimal hyperplasia & $13(18.6 \%)$ & $2(2.9 \%)$ & 0 & $<0.001$ \\
Any plaque & $18(25.7 \%)$ & $27(38.6 \%)$ & $19(27.1 \%)$ & NS
\end{tabular}

Conclusion: This study shows the high prevalence of asymptomatic atherosclerosis in RA and PsA compared to general population. Even though it was shown a higher prevalence of CP in PsA, subclinical atherosclerosis in RA patients may have an increased clinical significance. The presence of carotid plaque between groups was not statistically significant. We observed increased prevalence of carotid intimal hyperplasia in RA and PsA compared with age-matched controls. We emphasize the value of ultrasonography in the detection of early atherosclerosis lesions.

References:

[1] Wah-Suarez, M. I., et.al. (2019). Carotid ultrasound findings in rheumatoid arthritis and control subjects: A case-control study. International Journal of Rheumatic Diseases, 22(1), 25-31.

Disclosure of Interests: None declared

DOI: 10.1136/annrheumdis-2020-eular.5197 